area, each of whom has made substantial contributions to their respective fields, Michael Pender (largely on CNS disorders), and Pamela McCombe (more peripheral). A sound, broad, and welcome introduction (in part by Ian Frazer, Professor of Medicine in Queensland) is followed by chapters on multiple sclerosis, and on acute disseminated encephalomyelitis. Progressing down the neuraxis to the Guillain-Barre syndrome and CIDP, myasthenia and muscle disease, the book ends with a single chapter on paraneoplastic disorders, and one on connective tissue disease/vasculitis. And it is a far from superficial jaunt through these disparate but related topics-laboratory studies are rightly emphasised, with a 60 page chapter on experimental allergic encephalomyelitis, and a total of 125 pages specifically addressing experimental models or basic principles of neuroimmunology. The book is excellently and comprehensively referenced, over a third of its 350 pages comprising bibliography. Sadly, there is not a single illustrationbut perhaps grown ups do not need pictures. This is a well-written book, a good read accurate and up-to-date, and a timely review of an increasingly important field. NEIL SCOLDING

Introduction to the Neurologic Examination. By MICHAEL F NOLAN. (Pp $224 ; £ 18 \cdot 00$.) Published by Waverly Europe Ltd, London 1996. ISBN 0-8036-0017-8.

The concept behind this book is important: the marriage of clinical examination with neuroanatomical understanding, a formidable task, bearing in mind the end result of most preclinical courses. Does the author succeed? Almost. Certainly he has the correct credentials: associate professor of both anatomy and neurology; and the correct philosophy: "to make the neurologic evaluation a reasoned, purposeful, and dynamic series of interactions between clinicians and patients."

Central to this thesis is the careful use of line diagrams drawn from Neuroanatomy: $A n$ Atlas of Structures, Sections, and Systems, by DE Haines. These are clearly laid out in a schematic $2 \mathrm{D}$ form, demonstrating the routes of the appropriate pathways, which allow navigation even in the thickest neuronal jungle.

After a standard opening set on history taking, this book unfolds with the menta status examination incorporating helpful sections on gnosis and praxis. Yet, I think that it is at its best describing the functional anatomy of the brainstem. The demonic complexities of the trigeminal nerve demonstrates this well. Sensory and motor path are followed from ganglia to nuclei to thalamus and cortex with an emphasis on topographical representation and clinical examination. It does not attempt to describ specific lesions, but uses juxtaposition of the neurological/anatomical domains to enable real awareness to develop. Likewise the lower "Cinderella" cranial nerves are given equal prominence, with useful hints on bulbar examination.

Limb examination divides into motor reflex, and sensory systems as expected, with discussion of muscle spindles, sensory transduction, and central relay nuclei; as well as the standard pathways by which the messages are routed. I was glad that the author stresses reflex reinforcement and the diagram of reflex man was a clever idea. However, a 6 point reflex grading scheme seemed a little extreme even for the most careful examiner!

The book ends with a chapter on recording and presenting data, which although useful for placing neurological history taking and examination in overall context is unnecessary for the audience which I think this book would best serve. Probably the balance for the peri-MRCP clinician, this book is too closely packed for a student on a four week attachment. Why does this book only partially succeed in its laudable aims? I think that to really form a sound neuroanatomical base for clinical application, more pages of words would have to be replaced with more pages of diagrams. A little more Haines and a little less Nolan.

\section{JEREMY CHATAWAY}

Oppenheimer's Diagnostic Neuropathology. Edited by MARGARET M ESIRI. (Pp 458; £125.00.) Published by Blackwell Science, Oxford: 1996. ISBN 0865429154.

Diagnostic Neuropathology: a practical manual is now available in a new edition. Dr Margaret Esiri has undertaken this task single handedly, following the death of the book's coauthor David Oppenheimer. The text of this second edition is altered only to include new information in neuropathology. Actually, no other change was necessary. The late Lucien Rubinstein recognised this when he wrote, in the forward to the first edition, that it was "one of those rarer books which fall between the two extremes (of thin and lean or fat and crammed) and in which the balance between the skeletal outline and a profusion of details has been carefully adjusted, and the mixture blended with art". Yet, this new edition is not merely updated. It is immensely improved by the production of most illustrations in full colour and by the introduction of numerous illustrations of magnetic resonance images (MRI) to correspond with those of pathological specimens.

The quality of illustrations is superb, ranking with that found in sophisticated atlases of pathology. There is scarcely a page which is not graced by one or more photographs of a whole brain, brain slices, wet smears of biopsy material, histological sections, muscle histochemistry, resin embedded nerve sections or an osmicated teased fibre preparation. A nine page atlas of brain slices in both coronal and horizontal planes is also offered; this consists of black and white photographs of brain slices, each of which is paired with a corresponding MR image.

As its title clearly indicates, the book is intended as a guide to neuropathological methods; it is aimed principally at apprentices in neuropathology and at general pathologists with an interest. The book achieves this with enviable clarity and in so doing it provides the ideal foundation for subsequent excursions into the weightie and more comprehensive tomes to be found on neuropathology shelves. The neuropathology trainee will benefit from chapters which offer guidance on the neuropathological necropsy and the dissection and sampling of the brain and spinal cord. Especially useful is a chapter which indicates how one should proceed at necropsy in the case of a particular neurological sign or symptom; for instance a case of ataxia, autonomic failure ophthalmoplegia, involuntary movement, Horner's syndrome, disorders of speech and language and so on.
However, those called to the neuropathological "priesthood" are few, so the success of the first edition of Oppenheimer and Esiri's book is evidence that it has a much wider readership. Participants in a modern clinical neuroscience service must know the rudiments of specialties other than their own. Neuropathologists must have some knowledge of clinical neurology, neurosurgery, and neuroimaging in order to give the best possible neuropathological opinion. Similarly, trainees and specialists in clinical neurology, neurosurgery, and neuroradiology require knowledge of the principles of diagnostic neuropathology if they are to understand the role of surgical biopsy and neuropathological necropsy in clinical practice. In my opinion, the new edition of Diagnostic Neuropathology offers them the best means with which to acquire this essential perspective.

General pathologists will also find much in this book that is pertinent to their practice. Injuries to the head and spinal column, intracranial haemorrhage, cerebral infarction, and hypoxia are encountered almost daily in the mortuary. This book will enable them to deal competently with straightforward problems and to recognise those cases which require referral of nervous tissues to their neuropathological colleagues.

Lucien Rubinstein said of this book that it "exudes the pleasure of problem-solving and breathes the joy of neuropathology". That endorsement is fully deserved.

JOHN XUEREB

Localisation in Clinical Neurology. Edited by JOSEPH C MASDEU AND JOSE BILLER. (Pp 384; $£ 84.00$ HB). Published by Churchill Livingstone, Edinburgh: 1996. ISBN 0316099929.

Many neurologists and students of neurology will be familiar with this book, as the first two editions of it appeared in 1985 and 1990 respectively. The book concentrates on the anatomy and clinical signs of lesions at various levels of the nervous system. The book starts in the peripheral nervous system and then progresses in subsequent chapters up the neural axis. Thus it starts with the peripheral nerves and in the following chapters concentrates on the cervical, brachial and, lumbrosacral plexuses before confronting the spinal nerves and roots and entering the spinal cord. On reaching the brainstem, the cranial nerves are encountered, and 233 pages are devoted to them highlighting their importance in neurological practice. The conclusion of these chapters heralds entry into the latter half of the book and more rostral structures, including a whole chapter on the much neglected thalamus. The book then concludes with a discussion on the localisation of lesions in comatosed patients, a situation in which the previous 20 chapters cannot be applied.

This book therefore forms a complete textbook for the examination and interpretation of signs and symptoms affecting both the central and peripheral nervous system. There are, however, no specific chapters devoted to the neuromuscular junction or muscle and there is little discussion of neuropsychiatry. These are, though, minor omissions in a book that is otherwise awesome in its coverage. A book that provides an invaluable and in depth discussion and explanation on everything you ever needed 